\title{
Exogenous Antioxidants are Double-edged Swords
}

\author{
Abdürrahim KOÇYiĞíT, Şahabettin SELEK
}

Department of Medical Biochemistry, Bezmialem Vakıf University School of Medicine, İstanbul, Turkey

\section{ABSTRACT}

Oxidant-antioxidant balance is crucially important in maintaining healthy biological systems. Under physiological conditions, the human antioxidative defense system, including superoxide dismutase (SOD), catalase (CAT), glutathione peroxidase (GSH-Px), and glutathione (GSH), allows the elimination of excess reactive oxygen species (ROS), including superoxide anions $\left(\mathrm{O}_{2}\right)$, hydroxyl radicals (HO), and alkoxyl radicals (RO). In addition, exogenous compounds with reducing capacity, such as vitamin C, vitamin E, carotenoids, and polyphenols are also essential for intact functioning of endogenous antioxidant defense system. Therefore, there is continuous demand for exogenous antioxidants to prevent oxidative stress. However, higher doses of isolated compounds could act as pro-oxidant either by presenting direct pro-oxidant behavior or by interfering with the normal doses of ROS that is required for normal cell functioning. In this review, synthetic antioxidants among supplementary antioxidants, fruit-rich nutrition and various supplementary products to strengthen our body will be considered and discussed under the light of experimental and epidemiological evidence. Antioxidants in physiological dose ranges are considered to be safe in healthy people and overdoses cause damage via pro-oxidative effects; therefore, the importance of doses of antioxidants, the lower preventive doses that protect healthy individuals from illnesses, and higher therapeutic doses that treat cancer patients will be emphasized in this review.

Keywords: Antioxidants, oxidative stress double-edged effects, phytoterapy

\section{Introduction}

\subsection{Free Radicals and Antioxidants}

Free radicals are high-energy and non-stable compounds containing electrons that do not form one or more pairs in their external atomic orbitals. They are formed by removing an electron from a non-radical atom or molecule or by adding an electron to an atom or molecule (1). The resulting radicals are very reactive and unstable. Because they can give electrons to other molecules or take electrons from them, they have reductive or oxidizing effects in the body. Reactive oxygen species (ROS), which make up a significant proportion of free radicals, are characterized by an oxygen atom with an electron that is unshared in the external orbit of the free radicals (2). Humans live in the presence of a wide range of environmental stressors that increase the body ROS production, such as ultraviolet (UV) radiation, microbes, allergens, increased ozone, cigarette smoke, and polycyclic aromatic hydrocarbons (3). Free oxygen radicals can often initiate many new free radical-forming chain reactions with a non-radical (oxygen-centered free radical) unpaired electron such as superoxide anion $\left(\mathrm{O}_{2}^{-}{ }^{-}\right.$), hydroxyl radical ( $\mathrm{HO}$. ), alkoxyl radical (RO), peroxyl radical (ROO), hydrogen peroxide $\left(\mathrm{H}_{2} \mathrm{O}_{2}\right)$, hypochlorous acid $(\mathrm{HOCl})$, and singlet oxygen $\left({ }^{1} \mathrm{O}_{2}\right)$, or ROS, which can be defined as an electronfree oxygen intermediate product carrier metabolite, oxidizes other compounds and can initiate free radical-forming chain reactions by converting them into free radicals (4). Partially stabilized radicals such as urate radical $\left(\mathrm{UrH}^{-}\right)$, ascorbyl radical (Asc'), vitamin E radical (VE), and phenoxy radical ( $\mathrm{PhI}$ ), which can be formed in an in vivo environment, and radicals such as reactive radicals (lipid radicals) and sulfur-centered radicals [e.g., glutathiyl radicals (GS)], which include carbon-centered free radicals and are formed in an aerobic environment, have high oxidative potential (2). The radical chain reaction typically continues until the anaerobic medium or substrate decreases. However, chain reac- 
tions can be stopped in the presence of non-radical product formation from two radicals or in the presence of chainbreaking antioxidants (VE, polyphenols) (5). Acute aerobic, anaerobic, and heavy exercise can result in excess reactive oxygen production (6). In this regard, $\mathrm{O}_{2}^{-}$resulting from the monoelectronic reduction of oxygen is described as the precursor molecule of ROS (7).

Experimental animal studies have shown that the production of toxic oxygen metabolites is stimulated in stressful situations such as immobilization stress, sleep deprivation, and stimulated exercise (8). Diets with excess fat and carbohydrate content and with partially low levels of antioxidant vitamins may also result in the overproduction of oxygen-derived radical products (9). Oxidative stress originating from ROS increases and antioxidant activity decreases in other conditions such as metabolism of alcohol or pharmacological agents, tobacco, radiation (X-ray) for therapy, hypothermia, inflammation, and excessive iron loading. Therefore, chronic diseases such as cardiovascular diseases (CVD), cancer, and neurodegenerative diseases have been associated with ROS causally or as a risk factor (11).

Antioxidants such as vitamin $\mathrm{C}$ and VE, carotenoids, and polyphenols that are taken exogenously are needed to balance our oxidant/antioxidant system because our endogenous antioxidant system is often inadequate to neutralize ROS during mitochondrial respiration and during normal cellular metabolism using molecular oxygen such as $\mathrm{NADPH}$ oxidase and xanthine oxidase (11). However, in contrast to what is expected, high doses of antioxidants generally have an effect on enhancing oxidative damage through pro-oxidant activity (12). The review focuses on the two-sided adverse effects of natural antioxidants. The relationship between the type, dose, and type of intake of exogenous antioxidants and beneficial effects will be discussed and the studies that show the two-sided adverse effects of exogenous antioxidants and the importance of the dose taken during the illness and during good health will be mentioned.

\section{Evidences of Preclinical and Clinical Investi- gations}

2.1. In Vivo Evidences Related to Exogenous Antioxidants In recent years, the intensive appearance of the programs related to the role of antioxidants in human life and health in the media has increased their popularity in the public. Compared with oxidizable compounds (DNA, proteins, lipids or carbohydrates), antioxidants are defined as molecules that have low concentrations and that delay or prevent the oxidative damage caused by ROS (13). Exogenous antioxidants play a key role in the delicate balance between oxidants and antioxidants in living organisms (14). While high doses of ROS cause pathophysiological changes, low amounts are required for normal physiological functions such as those in the immune defense system, vascular tonus, and signal transduction (15).
Living organisms have an antioxidant defense system (enzymatic and nonenzymatic) such as superoxide dismutase (SOD), glutathione peroxidase (GSH-Px), GSH, catalase (CAT), and bilirubin as well as exogenous antioxidants such as vitamin $\mathrm{C}$, $\mathrm{VE}$, and carotenoids that are mainly based on the diet (16). As in case of the regeneration of VE by GSH or as case of the in the inhibition of lipid peroxidation by vitamin $\mathrm{C}$, endogenous and exogenous antioxidants work synergistically to provide redox balance (17). While VE clears lipid peroxyl radicals (LOO) as a hydrogen donor in order to stop the free radical chain reaction, ascorbic acid rapidly reduces the $\alpha$-tocopherol radical and LDL to $\alpha$-tocopherol in membranes and probably stops $\alpha$-tocopherol radical-mediated damage (18).

In fact, people have to take vitamins through nutrients because they cannot synthesize them, and plant foods (apple, banana, tomato, potato, onion, broccoli, and others) are the natural sources of antioxidants (19). The main antioxidant compounds found in plant foods are phenolic or polyphenolic compounds in addition to vitamins. In particular, flavonoids commonly found in fruits and vegetables are molecules with strong antioxidant properties. Evidence related to in vitro studies demonstrates that plant-derived molecules such as polyphenolic compounds have antigenotoxic and anticytotoxic effects on oxidative stress-exposed cells (20).

Although antioxidant activities are well known, exogenous antioxidants also show pro-oxidant activity at high doses and particularly in the presence of metal ions such as iron and copper (21). Pro-oxidant activity is related to the molecular structure and concentrations of antioxidants (22). In cell modeling studies that have recently been conducted, pro-oxidative effects of some polyphenolic compounds such as quercetin, catechin, and gallic acid, which are known as antioxidants, have also been revealed (23). For example, in a study conducted using flavonoids (quercetin and fisetin), it has been reported that although flavonoids prevented cell death at low concentrations (10-20 mM), they caused cell death, DNA damage, and apoptosis at high concentrations (50-250 mM) in H4IIE cells (24).

In addition to high concentrations of antioxidants, the presence of metal ions has been reported to play an important role, and epigallocatechin (EGCG) has been shown to cause oxidative damage in isolated cellular DNAs in the presence of metal ions (25). In plants capable of reduction and chelation, nutritional antioxidants such as polyphenols show pro-oxidative activity in the presence of metal ions such as iron and copper, which are transition metals (26). The first mechanism of the antioxidative properties of natural compounds is to give hydrogen atom (-RH) or electron (-R) to the free radical (-R) with a direct effect. The second mechanism is as follows: when the antioxidant absorbs UV radiation or enters the antioxidation reaction as the chelator of transition metal ion, it acts as a singlet oxygen $\left({ }^{1} \mathrm{O}_{2}\right)$ activator or converts the hydroperoxide (ROOH) into nonradical products (27). However, antioxidants show the most 
reductive power with the Fenton reaction in the presence of metal ions, particularly $\mathrm{Fe}^{3+}$ and $\mathrm{Cu}^{+}$ions, and by increasing the formation of $\mathrm{OH}$, which is a potentially quite harmful radical among peroxides (28).

\section{Antioxidant $(\mathrm{AH})+\mathrm{Fe}^{3+}\left(\right.$ or $\left.\mathrm{Cu}^{+}\right) \longrightarrow \mathrm{A}+\mathrm{Fe}^{2+}\left(\right.$ or $\left.\mathrm{Cu}^{+}\right)+\mathrm{H}^{+}$ $\mathrm{H}_{2} \mathrm{O}_{2}+\mathrm{Fe}^{2+}($ or $\mathrm{Cu}+) \longrightarrow \mathrm{OH}+\mathrm{Fe}^{3+}\left(\right.$ or $\left.\mathrm{Cu}^{2+}\right)$}

When the antioxidant polyphenolic compounds clear free radicals, they turn into less reactive PhIs that are stabilized by the delocalization of unpaired electron around the aromatic ring (25). It is considered that the pro-oxidant activities of flavonoids of polyphenolic compounds are directly related to the number of $\mathrm{OH}$ groups present in the molecule (29). While the pro-oxidant activities of mono- and dihydroflavonoids cannot be shown, flavonoids that are in the B-ring and contain more than three $\mathrm{OH}$ groups have been shown to significantly increase the $\mathrm{OH}$ production in Fenton reaction (30). The pro-oxidant functions of flavonoids may also suppress their other beneficial effects. For example, the apoptosis and antibacterial effects of EGCG gallate depend on the conversion of $\mathrm{O}_{2}^{--}$to $\mathrm{H}_{2} \mathrm{O}_{2}$ by them (31). In reference to recent studies, the fact that high doses of diet-induced polyphenols lead to mitochondrial dysfunctions and apoptosis due to their pro-oxidant activity supports the explanation of possible phytotherapeutic anticancer mechanisms (25).

In addition to their antioxidative properties, the beneficial or harmful effects of natural antioxidant compounds depend on inflammation, nitrosative stress due to the overproduction of reactive nitrogen products, and carbonyl stress due to the excessive accumulation of reactive carbonyl products (32). In addition to the biphasic effects of antioxidants on oxidative metabolism, natural compounds may have opposite effects on inflammatory reactions. For example, while ß-carotene shows low-dose antioxidant (2) and antiinflammatory effects on HL-60 cells, it has been reported to have a pro-inflammatory effect at high doses by increasing the production of tumor necrosis factor alpha (TNF- $\alpha$ ) and interleukin-8 (IL-8), which are pro-oxidant and pro-inflammatory mediators (33). Regardless of antioxidant activity, by interacting with the intracellular signal cascade in cells and binding to many ATP-binding sites such as mitogen-activated protein kinase (MAPK) or mitochondrial ATPase, calcium plasma membrane ATPase, and protein kinase A, polyphenols may also activate the pathways and show modulatory effects. The ability of certain antioxidants such as quercetin and naringenin to inhibit cytochrome P450 enzymes that include bioactivation of chemical carcinogens constitutes the other chemopreventive mechanism of polyphenols against the development of cancers, including lung cancer (35).

\subsection{In Vivo Evidences Related to Exogenous Antioxidants} Experimental animal studies include epidemiological evidences and human studies. In animal studies, long-term intake of natural foods such as apples, olives, and honey has been shown to cause some adverse effects related to aging, including oxidative stress in the brain, mental disorders, and anxiety (36). Potential side effects of exogenous antioxidants on healthy humans have been first revealed by demonstrating the carcinogenic and toxic effects of synthetic antioxidants such as butylated hydroxyanisole (BHA) and butylated hydroxytoluene (BHT) on rodents and apes at high concentrations, possibly with a pro-oxidative effect. Surprisingly, in animal models, BHA has been reported to exhibit anticarcinogenic activity against different carcinogens (37). These different findings may be explained by the dose administered during treatment. In a certain part of the world, humans take $0.1 \mathrm{mg} / \mathrm{kg}$ of BHA and BHT per day. The $\mathrm{LD}_{50}$ dose of these synthetic antioxidants has been reported to be $2 \mathrm{mg} / \mathrm{kg}$ in several animal studies, and its toxicity on human health has been increasingly confirmed in cases of chronic intake. Interestingly, the presence of antioxidants such as BHT and BHA in foods at high concentrations may also increase their deterioration depending on their pro-oxidant activity, rather than increasing their shelf life (38).

In relation to humans, the healthy functions of dietary ingredients, such as antimutagenic, anticarcinogenic, and antiaging effects, are discussed in relation to their antioxidant properties (14). Epidemiological studies investigating the preventive effects of natural antioxidant-rich diets on disease progression have played a key role in researches (35). In fact, considering that the development of chronic diseases such as CVD associated with oxidative stress is prevented by plant-derived foods, researches have been conducted on the assumption that regular intake of fruits and vegetables is related to a long life span (39). It has been found that long-term regular intake of vegetables and fruits reduces the risk of coronary artery disease and there is a negative correlation between high intake of vegetables and fruits and ischemic and hemorrhagic stroke (40). However, an increasing number of evidences suggest that the beneficial effects of plant foods are due to the synergistic effects of the complex mixture found in the whole of fruits and vegetables rather than the specific compounds that they contain (41). Epidemiological and observational studies conducted previously suggested that increasing the intake of carotenoid reduces the risk of development of gastrointestinal or lung cancer and the risk of CVD. However, one study showed that $B$-carotene intake does not alter the incidence of nonmelanoma skin cancer, while another study showed that $B$-carotene intake in smokers is not useful in reducing the incidence of cancer and can even increase the harmful effect (41).

\subsection{Exogenous Antioxidants and Cancer}

Despite intensive researches on the prevention of cancer formation in recent years, it is observed that the incidence of cancer is increasing worldwide. Although the intake of low-fat and high-antioxidant fiber foods is highly recommended, the incidence of cancer has not yet been reduced. One of the reasons may be that the manner and dose of intake of exogenous 
antioxidants are not well known in the prevention of cancer. In addition to studies showing the beneficial effects of antioxidants, there are studies suggesting that they have no effect or have harmful effects that increase the incidence of cancer (42). Although some studies on cancer and antioxidants have shown a negative association between the risk of cancer and antioxidant-rich nutrition and low-fat and high-fiber diets, some studies have shown that a high intake of antioxidants through the diet increases the risk of cancer (43). High-dose exogenous antioxidants are thought to increase the risk of cancer by causing an increase in ROS with a pro-oxidative effect. High doses of antioxidants selectively kill cancer cells also because of pro-oxidative effects. Because the endogenous ROS production is high due to the high metabolic activity of cancer cells, they can cause selective damage and death of cancer cells with a cumulative effect, along with the pro-oxidant activity caused by high-dose antioxidants (44). Therefore, it is very important to determine the preventive and therapeutic doses of antioxidants. Preventive dose can be defined as the dose that does not affect the growth of cancer or normal cell or prevents cancer formation. Therapeutic dose is the dose that kills cancer cells (45). For example, while the daily preventive dose to be taken in an adult human who is at a high risk of cancer is up to $500 \mathrm{IU}$ of vitamin $\mathrm{A}$, up to $2 \mathrm{~g}$ of vitamin C, up to $25 \mathrm{mg}$ of carotenoids, and up to $200 \mathrm{mg}$ of selenometinine, the therapeutic dose that is required to be taken by cancer patients who are under standard treatment or who do not receive any treatment should be $25,000 \mathrm{IU}$ or more of vitamin A, $10 \mathrm{~g}$ or more of vitamin C, $100 \mathrm{mg}$ or more of carotenoids, and $300 \mathrm{mg}$ or more of selenomethionine (45).

Some experiments have shown that antioxidants can enhance the antiproliferative effect of ionizing radiation and chemotherapeutics with a synergistic or additive effect in cancer cells and in an in vivo environment (46). In these studies, it has been shown that high-dose antioxidants can enhance the efficacy of standard chemotherapy by reducing their toxicity to improve tumor response. The selective effects of high-dose antioxidants on tumor cells are not fully understood. However, high-dose antioxidants should be specified as therapeutic doses. Although it has been found that a high dose of antioxidants alone or in combination with standard therapy selectively kills cancer cells and a low dose of antioxidants increases the proliferation of cancer cells and lowers the efficacy of radiation and chemotherapeutic treatment, it has been shown that high doses of the same antioxidants inhibit the proliferation of some cancer cells (47).

While some oncologists warn their patients to not take antioxidants during radiation therapy or chemotherapy, some oncologists recommend multivitamin preparations containing low-dose antioxidants even after the treatment (45). These recommendations may be wrong because cancer cells, like normal cells, need micronutrients containing antioxidants in order to reproduce and survive. Low doses of dietary antioxidants may stimulate the proliferation of some cancer cells (48).
Therefore, the use of multivitamin preparations containing low doses of antioxidants after treatment may be a risk factor to increase the recurrence of primary tumors by stimulating primary cancer cell residuals in some cancer patients. However, $60 \%$ of cancer patients receive antioxidant supplementation without the knowledge of oncologists, and most of them combine it with standard therapy. This can be detrimental by interfering with the efficacy of standard therapy because many multivitamin preparations contain antioxidants at preventive doses. Low doses of vitamins such as VE and vitamin C, selenium, retinol, and b-carotene can reduce cytotoxic effects by protecting cancer cells against free radical damage caused by chemotherapeutic agents or radiation used in standard therapy (49). Most of the time, neither oncologists nor patients are well informed about the potential danger of receiving antioxidants at low preventive doses during radiation or chemotherapy (45). Therefore, if antioxidants are to be used in combination with standard therapy in cancer patients, the following points should be considered:

1. The response of tumor cells and cancer cells to antioxidant doses is different.

2. The effects of preventive and therapeutic doses of antioxidants on cancer cells are different.

3. Therapeutic doses of antioxidants reduce not only the proliferation of tumor cells but also the toxicity of standard treatments and enhance the efficiency of the treatment.

4. While the preventive doses of antioxidants stimulate the proliferation of cancer cells, they may reduce the efficacy of standard therapy.

5. Multiple antioxidants are more effective than a single type of antioxidant.

6. For therapeutic doses of antioxidants to be effective, therapy be started 3-5 days before standard therapy, given twice daily, and continued throughout the treatment pe$\operatorname{riod}(45)$.

Therapeutic doses of antioxidants cause effects such as those on gene expression, protein levels and translocations, proliferation inhibition, and apoptosis in cancer cells according to the form and type of the antioxidant, treatment protocol, and tumor type. Combined use of more than one antioxidant in treatment can cause additive or synergistic effects. It has also been shown that the use of multiple antioxidants in therapeutic doses in cancer patients is more effective than the use of a single type of antioxidant and that the therapeutic doses of antioxidants increase the efficacy of chemotherapy and radiotherapy used in standard therapy (50).

\section{Conclusion}

Free radicals are chemical products that carry an unshared electron in the outer orbit. Free radicals are produced in animals and plants during aerobic metabolism and can cause cell death in mammals and plants because of lipid peroxidation due to oxidative stress and can cause protein and DNA damage or chronic diseases such as CVD and cancer. 
Because the antioxidant enzymes and other molecules produced endogenously are insufficient to prevent the oxidative damage caused by free radicals, the antioxidants taken exogenously are useful in protecting against free radicals and play an important role in preventing many diseases and cancer in humans. However, a high dose of exogenous antioxidants can cause chronic diseases such as cancer by causing pro-oxidantinduced oxidative damage. Thus, while exogenous antioxidants reduce oxidative stress at low doses, which are preventive doses, they can enhance the selective death of cancer cells and the effectiveness of standard therapy by increasing ROS production with a pro-oxidative effect at high doses, which are therapeutic doses.

It should be noted that antioxidant-containing foods and phytotherapeutic agents containing antioxidants that are commonly used worldwide, in particular, may have opposite effects like two-sided sharp swords. It should be kept in mind that every phytotherapeutic agent commonly used in conventional medicine should be used aiming at the target, as in synthetic medicines, after the doses to be used in the prevention of disease or in the treatment of chronic diseases such as cancer are determined by preclinical and clinical studies; otherwise, it may cause serious damage instead of benefit.

Author Contributions: Concept - A.K.; Design - A.K.; Supervision - A.K., Ş.S.;Funding- A.K., Ş.S.; Materials - A.K., Data Collection and/or Processing - A.K., Ş.S.; Analysis and/or Interpretation - A.K., Ş.S., ; Literature Review A.K., Ş.S.; Writing- A.K.; Critical Review - Ş.S.

Conflict of Interest: No conflict of interest was declared by the authors.

Financial Disclosure: The authors declared that this study has received no financial support.

\section{References}

1. Flora $S$. Role of free radicals and antioxidants in health and disease. Cell Mol Biol (Noisy-le-grand) 2007; 53: 1-2.

2. Valko M, Leibfritz D, Moncol J, Cronin MT, Mazur M, Telser J. Free radicals and antioxidants in normal physiological functions and human disease. Int J Biochem Cell Biol 2007; 39: 44-84. [CrossRef]

3. Pryor WA, Stone K. Oxidants in cigarette smoke radicals, hydrogen peroxide, peroxynitrate, and peroxynitritea. Ann N Y Acad Sci 1993; 686: 12-27. [CrossRef]

4. Pham-Huy LA, He H, Pham-Huy C. Free radicals, antioxidants in disease and health. International journal of biomedical science: IJBS 2008; 4: 89.

5. Rice-Evans CA, Miller NJ, Paganga G. Structure-antioxidant activity relationships of flavonoids and phenolic acids. Free radical biology and medicine 1996; 20: 933-56. [CrossRef]

6. Rosa EF, Takahashi S, Aboulafia J, Nouailhetas VL, Oliveira MG. Oxidative stress induced by intense and exhaustive exercise impairs murine cognitive function. Journal of neurophysiology. 2007; 98: 1820-6. [CrossRef]

7. Bouayed J. Polyphenols: a potential new strategy for the prevention and treatment of anxiety and depression. Current Nutrition \& Food Science 2010; 6: 13-8. [CrossRef]

8. Kumar A, Goyal R, Prakash A. Possible GABAergic mechanism in the protective effect of allopregnenolone against immobilization stress. European journal of pharmacology 2009; 602: 343-7. [CrossRef]

9. Bloomer RJ, Fisher-Wellman KH. Systemic oxidative stress is increased to a greater degree in young, obese women following consumption of a high fat meal. Oxidative medicine and cellular longevity 2009; 2: 19-25. [CrossRef]
10. Kocyigit A, Erel O, Gur S. Effects of tobacco smoking on plasma selenium, zinc, copper and iron concentrations and related antioxidative enzyme activities. Clinical biochemistry 2001; 34: 629-33. [CrossRef]

11. Halliwell B. Oxidative stress and neurodegeneration: where are we now? Journal of neurochemistry 2006; 97: 1634-58. [CrossRef]

12. Ullah M, Ahmad A, Khan HY, Zubair H, Sarkar FH, Hadi S. The prooxidant action of dietary antioxidants leading to cellular DNA breakage and anticancer effects: implications for chemotherapeutic action against cancer. Cell biochemistry and biophysics 2013; 67: 431-8. [CrossRef]

13. Sahiner UM, Cansin Sackesen M. Oxidative stress and antioxidant defense. 2012.

14. Pandey KB, Rizvi SI. Plant polyphenols as dietary antioxidants in human health and disease. Oxidative Medicine and Cellular Longevity 2009; 2: 270-8. [CrossRef]

15. Martin K, Barrett J. Reactive oxygen species as double-edged swords in cellular processes: low-dose cell signaling versus high-dose toxicity. Human \& experimental toxicology 2002; 21: 71-5. [CrossRef]

16. Andre CM, Larondelle Y, Evers D. Dietary antioxidants and oxidative stress from a human and plant perspective: a review. Current Nutrition \& Food Science 2010; 6: 2-12. [CrossRef]

17. Valko M, Leibfritz D, Moncol J, Cronin MT, Mazur M, Telser J. Free radicals and antioxidants in normal physiological functions and human disease. The international journal of biochemistry $\&$ cell biology 2007; 39: 44-84. [CrossRef]

18. Niki E, Noguchi N, Tsuchihashi $\mathrm{H}$, Gotoh N. Interaction among vitamin C, vitamin E, and beta-carotene. The American journal of clinical nutrition 1995; 62: 1322S-6S.

19. Manach C, Scalbert A, Morand C, Rémésy C, Jiménez L. Polyphenols: food sources and bioavailability. The American journal of clinical nutrition 2004; 79: 727-47.

20. Kocyigit A, Koyuncu I, Taskin A, Dikilitas M, Bahadori F, Turkkan B. Antigenotoxic and antioxidant potentials of newly derivatized compound naringenin-oxime relative to naringenin on human mononuclear cells. Drug and chemical toxicology 2015: 1-8.

21. Azam S, Hadi N, Khan NU, Hadi SM. Prooxidant property of green tea polyphenols epicatechin and epigallocatechin-3-gallate: implications for anticancer properties. Toxicology in vitro. 2004; 18: 555-61. [CrossRef]

22. Yen G-C, Duh P-D, Tsai H-L, Huang S-L. Pro-oxidative properties of flavonoids in human lymphocytes. Bioscience, biotechnology, and biochemistry. 2003; 67: 1215-22. [CrossRef]

23. Robaszkiewicz A, Balcerczyk A, Bartosz G. Antioxidative and prooxidative effects of quercetin on A549 cells. Cell biology international 2007; 31: 1245-50. [CrossRef]

24. Wätjen W, Michels G, Steffan B, Niering P, Chovolou Y, Kampkötter A, et al. Low concentrations of flavonoids are protective in rat H4IIE cells whereas high concentrations cause DNA damage and apoptosis. The Journal of nutrition 2005; 135: 525-31.

25. Galati G, O'Brien PJ. Potential toxicity of flavonoids and other dietary phenolics: significance for their chemopreventive and anticancer properties. Free Radical Biology and Medicine 2004; 37: 287-303. [CrossRef]

26. Decker A. Phenolics: prooxidants or antioxidants? Nutrition Reviews 1997; 55: 396-8. [CrossRef]

27. Gordon MH. The development of oxidative rancidity in foods. Antioxidants in food practical applications 2001.

28. Maisuthisakul P, Suttajit M, Pongsawatmanit R. Assessment of phenolic content and free radical-scavenging capacity of some Thai indigenous plants. Food chemistry 2007; 100: 1409-18. [CrossRef]

29. Cao G, Sofic E, Prior RL. Antioxidant and prooxidant behavior of flavonoids: structure-activity relationships. Free Radical Biology and Medicine 1997; 22: 749-60. [CrossRef]

30. Heim KE, Tagliaferro AR, Bobilya DJ. Flavonoid antioxidants: chemistry, metabolism and structure-activity relationships. The Journal of nutritional biochemistry. 2002; 13: 572-84. [CrossRef] 
31. Maeta K, Nomura W, Takatsume Y, Izawa S, Inoue Y. Green tea polyphenols function as prooxidants to activate oxidative-stress-responsive transcription factors in yeasts. Applied and environmental microbiology. 2007; 73: 572-80. [CrossRef]

32. Rahman I, Biswas SK, Kirkham PA. Regulation of inflammation and redox signaling by dietary polyphenols. Biochemical pharmacology. 2006; 72: 1439-52. [CrossRef]

33. Yeh S-L, Wang H-M, Chen P-Y, Wu T-C. Interactions of $\beta$-carotene and flavonoids on the secretion of pro-inflammatory mediators in an in vitro system. Chemico-biological interactions 2009; 179: 386-93. [CrossRef]

34. Williams RJ, Spencer JP, Rice-Evans C. Flavonoids: antioxidants or signalling molecules? Free Radical Biology and Medicine 2004; 36: 83849. [CrossRef]

35. Le Marchand L, Murphy SP, Hankin JH, Wilkens LR, Kolonel LN. Intake of flavonoids and lung cancer. Journal of the National Cancer Institute 2000; 92: 154-60. [CrossRef]

36. Chepulis LM, Starkey NJ, Waas JR, Molan PC. The effects of longterm honey, sucrose or sugar-free diets on memory and anxiety in rats. Physiology \& behavior 2009; 97: 359-68. [CrossRef]

37. King MM, McCay PB, Kosanke SD. Comparison of the effect of butylated hydroxytoluene on $\mathrm{N}$-nitrosomethylurea and 7, 12-dimethylbenz [a] anthracene-induced mammary tumors. Cancer letters 1981; 14: 219-26. [CrossRef]

38. Akoh CC, Min DB. Food lipids: chemistry, nutrition, and biotechnology: CRC Press; 2008. [CrossRef]

39. Gormaz JG, Quintremil S, Rodrigo R. Cardiovascular Disease: A Target for the Pharmacological Effects of Quercetin. Current topics in medicinal chemistry. 2015; 15: 1735-42. [CrossRef]

40. He FJ, Nowson CA, MacGregor GA. Fruit and vegetable consumption and stroke: meta-analysis of cohort studies. The Lancet 2006; 367: 3206. [CrossRef]
41. Liu RH. Health benefits of fruit and vegetables are from additive and synergistic combinations of phytochemicals. The American journal of clinical nutrition 2003; 78: 517S-20S.

42. Tong L, Chuang CC, Wu S, Zuo L. Reactive oxygen species in redox cancer therapy. Cancer Lett 2015; 367: 18-25. [CrossRef]

43. López-Lázaro M. Anticancer and carcinogenic properties of curcumin: considerations for its clinical development as a cancer chemopreventive and chemotherapeutic agent. Molecular nutrition \& food research 2008; 52: S103-S27. [CrossRef]

44. Leon-Gonzalez AJ, Auger C, Schini-Kerth VB. Pro-oxidant activity of polyphenols and its implication on cancer chemoprevention and chemotherapy. Biochem Pharmacol 2015. [CrossRef]

45. Prasad KN. Micronutrients in Health and Disease: CRC Press; 2010. [CrossRef]

46. Hosseini A, Shafiee-Nick R, Mousavi SH. Combination of Nigella sativa with Glycyrrhiza glabra and Zingiber officinale augments their protective effects on doxorubicin-induced toxicity in h9c2 cells. Iranian journal of basic medical sciences. 2014; 17: 993-1000.

47. Prasad KN, Hernandez C, Edwards-Prasad J, Nelson J, Borus T, Robinson WA. Modification of the effect of tamoxifen, cis-platin, DTIC, and interferon- $\alpha 2 \mathrm{~b}$ on human melanoma cells in culture by a mixture of vitamins. 1994.

48. Crowe DL, Kim R, Chandraratna RA. Retinoic Acid Differentially Regulates Cancer Cell Proliferation via Dose-Dependent Modulation of the Mitogen-Activated Protein Kinase Pathway11NIH grant DE10966. Molecular cancer research. 2003; 1: 532-40.

49. Akan Z, Garip AI. Antioxidants may protect cancer cells from apoptosis signals and enhance cell viability. Asian Pacific journal of cancer prevention: APJCP 2013; 14: 4611-4. [CrossRef]

50. Kumar B, Jha MN, Cole WC, Bedford JS, Prasad KN. D-alpha-tocopheryl succinate (vitamin E) enhances radiation-induced chromosomal damage levels in human cancer cells, but reduces it in normal cells. Journal of the American College of Nutrition 2002; 21: 339-43. [CrossRef] 